\title{
Control and in-situ imaging of heat \& gas mediated processes in FIB/SEM system
}

\author{
Libor Novák ${ }^{1}$, Miroslav Kolíbal ${ }^{2}$, Min Wu $^{1}$, Petr Wandrol $^{1}$, and Tomáš Vystavěl ${ }^{1}$ \\ 1. Thermo Fisher Scientific, Vlastimila Pecha 12, 62700 Brno, Czech Republic \\ 2. Central European Institute of Technology - CEITEC, Purkyňova 123, Brno, Czech Republic
}

During last years, in-situ imaging of chemical vapor deposition (CVD), reduction, and oxidation processes was performed in SEM on a sample heated to high temperature at the presence of reactive gas in the SEM chamber [1] [2]. Nevertheless, gas injection into the whole SEM chamber is limited by safety restrictions in case that an explosive or toxic gas is required. Moreover cleanness of the CVD process can be negatively influenced by contamination or oxidation from residual gases remaining in the large volume of the SEM chamber [3].

In this contribution we present hardware solution and experimental results of a new miniaturized reactor for in-situ SEM experiments, depicted in Figure 1. The design combines sample heating (up to $1200^{\circ} \mathrm{C}$ ) by a micro-heating-plate [4] with possibility for gas injection into a differentially pumped reaction space of low volume ( 2 microliters) surrounding the heated sample. Defined gas flow through the sample area, higher and stable pressure (up to $100 \mathrm{~Pa}$ ) achievable inside the miniaturized SEM reactor during in-situ experiments are clear benefits compared to gas injection from a needle. While the existing reactors of sub-microliter reaction space closed between two MEMS chips, which are dedicated primarily for gas and heat mediated TEM experiments, are limited to inspection of liquid samples or to imaging of submicron particles dissolved in a liquid carrier [5], design of the miniaturized SEM reactor provides possibility for processing of any bulk sample, which can be placed on the SEM stage.

During the sample preparation flow, upper side of the SEM reactor formed by a pressure limiting aperture (Figure 1) is removed and extracted part of the bulk sample can be placed to the micro-heatingplate using FIB milling and consequent transfer by using micromanipulator needle [6] (see Figure 2c). Before the gas assisted experiment is started, the pressure limiting aperture is mounted to close the reaction volume and assure desired overpressure in sample area. Gas flow through the hole in the pressure limiting aperture with a diameter of few hundreds of microns is low enough to enable high vacuum operation of the SEM chamber, which is required for ultrahigh resolution SEM imaging. Detection of secondary as well as backscattered electrons is possible, preferably using in-lens detectors, as both signal electrons can pass through the pressure limiting aperture. Optionally, simultaneous STEM imaging through thinned areas of the micro-heating-plate is possible.

Results of extended possibilities of in-situ SEM experiments, facilitated by miniaturized SEM reactor, are demonstrated in Figures $2 \mathrm{a}$ and $2 \mathrm{~b}$ showing growth of rutile $\left(\mathrm{TiO}_{2}\right)$ nanowires from titanium substrate [7]. The CVD process [8] performed at controlled environment stimulated by $900^{\circ} \mathrm{C}$ sample temperature and $100 \mathrm{~Pa}$ of acetone vapor in the reactor volume was imaged using a Thermo Fisher Helios G4 UX FIB/SEM system.

To demonstrate the stability of the processses in the miniaturized SEM reactor system we performed high resolution immersion mode observation of tungsten-oxide nanowires evolution from tungsten disulfide substrate exposed to the water vapor flow at $750^{\circ} \mathrm{C}$ (Figure 3). 
References:

[1] M Kolíbal et al, Nano Lett. 16 (8) (2016), 4880-4886

[2] Z-J Wang et al, ACS Nano 9 (2) (2015), 1506-1519

[3] P Roediger et al, J. Vac. Sci. Technol. B 27 (2009), 2711-2717.

[4] L Mele et al, Microsc. Res. Tech. 79 (2016), 239-250.

[5] X Zhang et al, Chem. Commun. 53 (2017), 13213-13216.

[6] L Novák et al, Microsc. Microanal. 22 (Suppl. S3) (2016), 184-185.

[7] https://www.youtube.com/watch?v=sWbVsmYPYlI\&feature=em-share_video_user

[8] X Peng et al, Nanotechnology 16 (2005) 2389-2395

[9] The authors acknowledge funding from Technology Agency of the Czech Republic, TE01020118

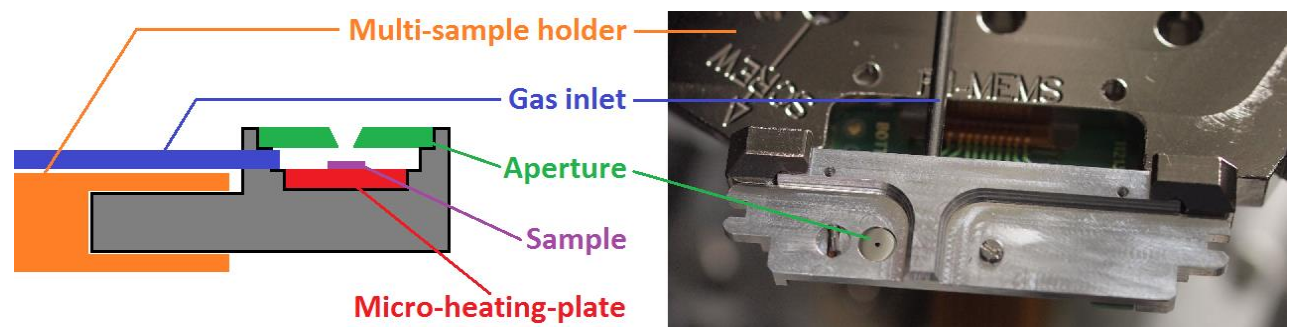

Figure 1. Miniaturized reactor connected to multi-sample holder of a SEM/FIB system, which assures heating control up to $1200^{\circ} \mathrm{C}$. Pressure limiting aperture (in green) separates the overpressure (up to 100 $\mathrm{Pa})$ inside the reactor from the high vacuum environment $(<1 \mathrm{E}-2 \mathrm{~Pa})$ of the SEM chamber.

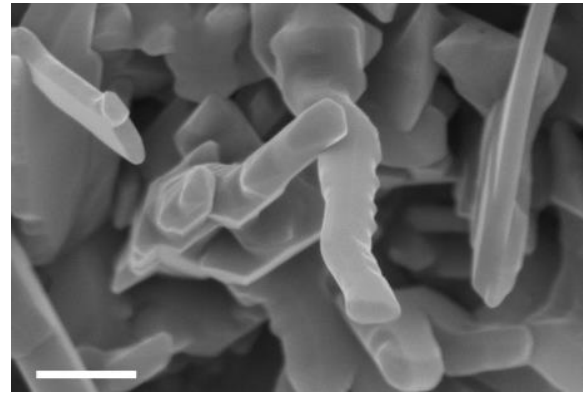

(a)

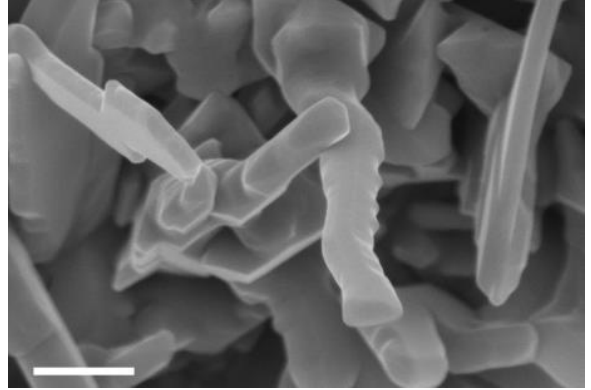

(b)

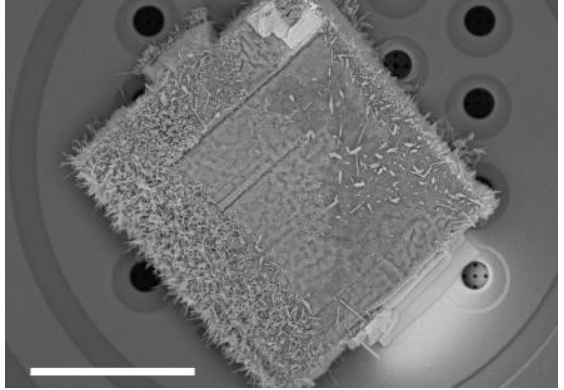

(c)

Figure 2. Evolution of rutile $\left(\mathrm{TiO}_{2}\right)$ nanowires in acetone vapor $(100 \mathrm{~Pa})$ environment at $900^{\circ} \mathrm{C}$; time step between (a) and (b) is 8 minutes. Titanium substrate was prepared by FIB milling and placed on the micro-heating-plate (c) using micromanipulator needle. Scale bar: $300 \mathrm{~nm}$ for (a) and (b); $30 \mu \mathrm{m}$ for (c).
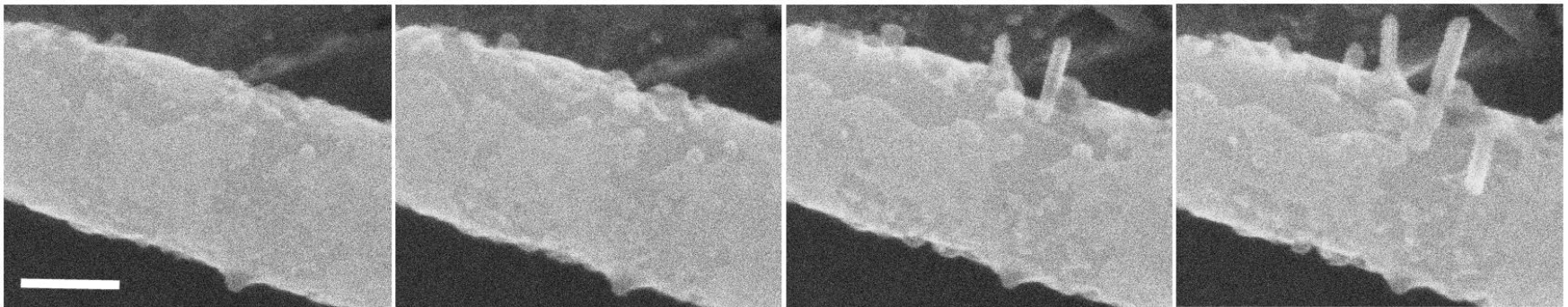

Figure 3. An image sequence ( 3 minute steps) taken during oxidation reaction $\left(725{ }^{\circ} \mathrm{C}, \mathrm{H}_{2} \mathrm{O}\right.$ vapor) of tungsten disulfide nanotube. Unusual behavior is observed, beginning with the formation of amorphous hillocks, followed by the growth of tungsten oxide nanowires. Scale bar: $100 \mathrm{~nm}$. Figures 2 and 3 were taken in high resolution immersion mode of the Thermo Scientific Helios G4 UX using in-lens SE detector (TLD). 